\title{
MINKOWSKI POINT SET COMBINATIONS
}

\section{Daniela VELICHOVÁ}

Institute of Mathematics and Physics, Faculty of Mechanical Engineering, Slovak University of Technology in Bratislava, Nám. Slobody 17, 81231 Bratislava, SR, daniela.velichova@stuba.sk

Keywords: Minkowski sum, Minkowski product, Minkowski point set combinations

Abstract Paper brings few ideas about a concept of Minkowski combinations of point sets, which can be defined as generalisation of set operations defined on point sets in the Euclidean space, Minkowski sum and Minkowski product. Various forms of Minkowski linear and matrix combinations of two point sets are introduced. Presented algebraic tool for modelling point sets determined by defined vector operations can be used in geometric modeling, in applied computer graphics, or for solving other problems in vector analysis and theory of vector fields related to applied mechanics and mechatronics.

\section{INTRODUCTION}

Operations of Minkowski sum and Minkowski product are point set operations defined on subsets of the Euclidean space $\mathbf{E}^{n}$, which are based on well defined operations on vectors, namely sum and exterior wedge product of two vectors in the associated vector field $V\left(\mathbf{E}^{n}\right)$. Minkowski sum of two point sets has been introduced by Hermann Minkowski in 1903. Nowadays this set operation has been re-introduced in connection to finding geometric algorithms for description of specific geometric problems dealing with layout optimisation, planning trajectory of a robot rigid motion in the working space avoiding obstacles, in offsetting and dense packing, for determination of equidistant manifolds and for shape modelling and morphing purposes in computer graphics. There exist more interpretations of Minkowski sum of two point sets, while the most common one is by means of vector sum of the position vectors of all points in the given sets.

Let $V\left(\mathbf{E}^{n}\right)$ be the vector space associated to the Euclidean space $\mathbf{E}^{n}$ with the Cartesian orthogonal coordinate system $\left\langle 0 ; \mathbf{e}_{1}, \mathbf{e}_{2}, \ldots, \mathbf{e}_{n}\right\rangle$ defined by the direction unit vectors of the coordinate axes $\mathrm{e}_{1}=(1,0, \ldots, 0), \mathrm{e}_{2}=(0,1,0 \ldots, 0) \ldots \mathrm{e}_{\mathrm{n}}=(0, \ldots, 0.1)$ that are forming the orthonormal basis $\left\{\mathbf{e}_{1}, \mathbf{e}_{2}, \ldots, \mathbf{e}_{n}\right\}$ of the vector space $V\left(\mathbf{E}^{n}\right)$.

Any two arbitrary vectors $\mathbf{u}, \mathbf{v} \in V\left(\mathbf{E}^{n}\right)$ can be given by their coordinates in this basis, where

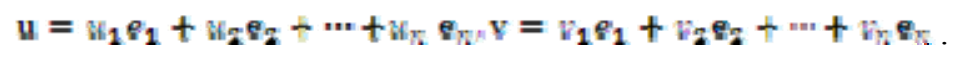

Sum of vectors $\mathbf{u}$ and $\mathbf{v}$ from $V\left(\mathbf{E}^{n}\right)$ is vector $\mathbf{u}+\mathbf{v}$ from the same vector space $V\left(\mathbf{E}^{n}\right)$ defined as

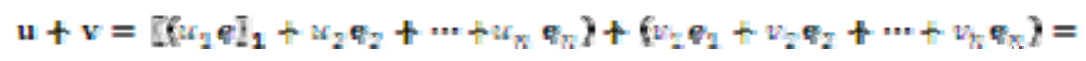

The following four properties are satisfied for vector sum in the vector space $V\left(\mathbf{E}^{n}\right)$. 
1. Commutativity: $\mathbf{u}+\mathbf{v}=\mathbf{v}+\mathbf{u}$.

2. Associativity: $(\mathbf{u}+\mathbf{v})+\mathbf{w}=\mathbf{u}+(\mathbf{v}+\mathbf{w})$.

3. Existence of neutral element, zero vector $0: \mathbf{u}+\mathbf{0}=\mathbf{u}, \forall \mathbf{u}$.

4. Existence of additive inverse vector $-\mathbf{u}$ for any vector $\mathbf{u}$ : $\mathbf{u}+(-\mathbf{u})=\mathbf{0}, \forall \mathbf{u}$.

Any point $m \in \mathbf{E}^{n}$ determined by an $n$-tuple of numbers that are its Cartesian coordinates $m=\left(m_{1}, m_{1}, \ldots, m_{n}\right)$ can be attached its position vector $\mathbf{m}$ by means of the following mapping

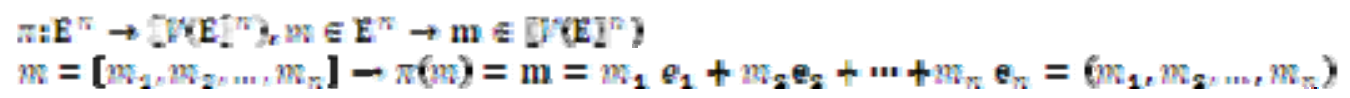

Denoting $V(A), V(B)$ sets of all position vectors of points from sets $A$ and $B$ respectively, the definition of Minkowski sum of the given point sets $A$ and $B$ can be precisely formulated by means of sum of position vectors of the respective points from these sets.

Definition 1. Sum of two points $a, b$ in the space $\mathbf{E}^{n}$ is such point $c \in \mathbf{E}^{n}$, whose position vector from the vector space $V\left(\mathbf{E}^{n}\right)$ is the sum of position vectors of points $a$ and $b$

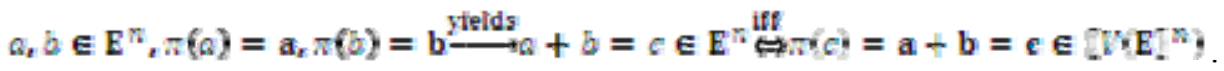

Definition 2. Minkowski sum of two different point sets $A$ and $B$ in $\mathbf{E}^{\mathrm{n}}$ is a point set in $\mathbf{E}^{\mathrm{n}}$, whose points are sums of all points from set $A$ with all points from set $B$, therefore it is point set

$$
A \oplus B=\{a+b: a \in A, b \in B\}, \text { while } A \oplus \varnothing=\varnothing .
$$

Definition of Minkowski product of two point sets $A$ and $B$ in the space $\boldsymbol{E}^{\mathrm{n}}$ can be precisely formulated by means of product of position vectors of the respective points in these sets. The concept of exterior, or wedge product of two vectors from $n$-dimensional vector space $V^{n}$ has been chosen for this definition. Exterior (wedge) product of vectors $\mathbf{u}, \mathbf{v} \in V^{n}$ is vector $\mathbf{u} \wedge \mathbf{v}$ from the vector space $W^{p}$ of dimension $p=\frac{n(n-1)}{2}$ determined as follows

$$
\begin{aligned}
& \mathbf{u} \Lambda \mathbf{v}= \\
& =\left(u_{1} \mathbf{e}_{1}+u_{2} \mathbf{e}_{2}+\ldots+u_{n} \mathbf{e}_{n}\right) \Lambda\left(v_{1} \mathbf{e}_{1}+v_{2} \mathbf{e}_{2}+\ldots v_{n} \mathbf{e}_{n}\right)= \\
& =\left(u_{1} v_{2}-u_{2} v_{1}\right)\left(\mathbf{e}_{1} \Lambda \mathbf{e}_{2}\right)+\ldots+\left(u_{1} v_{n}-u_{n} v_{1}\right)\left(\mathbf{e}_{1} \Lambda \mathbf{e}_{n}\right)+ \\
& +\left(u_{2} v_{3}-u_{3} v_{2}\right)\left(\mathbf{e}_{2} \Lambda \mathbf{e}_{3}\right)+\ldots+\left(u_{2} v_{n}-u_{n} v_{2}\right)\left(\mathbf{e}_{2} \Lambda \mathbf{e}_{n}\right)+ \\
& +\ldots+\left(u_{n-1} v_{n}-u_{n} v_{n-1}\right)\left(\mathbf{e}_{n-1} \Lambda \mathbf{e}_{n}\right)
\end{aligned}
$$

where vectors $\left\{\mathbf{e}_{1} \Lambda \mathbf{e}_{2}, \mathbf{e}_{1} \Lambda \mathbf{e}_{3}, \ldots, \mathbf{e}_{1} \Lambda \mathbf{e}_{n}, \mathbf{e}_{2} \Lambda \mathbf{e}_{3}, \ldots, \mathbf{e}_{2} \Lambda \mathbf{e}_{n}, \ldots, \mathbf{e}_{n-1} \Lambda \mathbf{e}_{n}\right\}$ form the orthonormal basis of the vector space $W^{p}$. For $n=3$ also $p=3$ and we receive the standard cross (vector) product of two vectors in space $V\left(\mathbf{E}^{3}\right)$. Wedge product of vectors from $V\left(\mathbf{E}^{4}\right)$ is vector in the 6 dimensional vector space $W^{6}$, while both vector spaces are orthogonal complements in space $W^{10}$.

Properties of wedge vector product are as follows:

1. Anti-commutativity: $\mathbf{u} \wedge \mathbf{v}=-(\mathbf{v} \wedge \mathbf{u})$

2. Distributivity with respect to scalar multiple of a vector and vector sum

$$
(\mathbf{u}+\mathbf{v}) \wedge \mathbf{w}=\mathbf{u} \wedge \mathbf{v}+\mathbf{v} \wedge \mathbf{w}
$$


For all $\mathbf{u} \in V^{n}$ and real constants $c_{1}, c_{2}$ holds:

$\left(c_{1} \mathbf{u}_{1}+c_{2} \mathbf{u}_{2}\right) \wedge \mathbf{v}=c_{1}\left(\mathbf{u}_{1} \wedge \mathbf{v}\right)+c_{2}\left(\mathbf{u}_{2} \wedge \mathbf{v}\right)$

$\mathbf{u} \wedge\left(c_{1} \mathbf{v}_{1}+c_{2} \mathbf{v}_{2}\right)=c_{1}\left(\mathbf{u} \wedge \mathbf{v}_{1}\right)+c_{2}\left(\mathbf{u} \wedge \mathbf{v}_{2}\right)$

3. $\mathbf{u} \wedge \mathbf{u}=\mathbf{0}$ for all $\mathbf{u}$

4. Product of two unit vectors is a unit vector: $\left|\mathbf{e}_{\mathrm{i}} \wedge \mathbf{e}_{\mathrm{j}}\right|=1, \forall i, j=1,2, \ldots, n$

\section{MINKOWSKI COMBINATIONS OF POINT SETS}

Concept of Minkowski linear combination of point sets is an analogy of the concept of linear combination of vectors. On the base of scalar multiple of a vector there can be introduced the concept of a scalar multiple of a point set used in definition of point set combination.

Definition 3. Multiple of a point set $A \subset \mathbf{E}^{n}$ by scalar $k \in R$ is a point set $A_{k} \subset \mathbf{E}^{n}$ with elements in all such points, whose position vectors are $k$-multiples of position vectors of all points from $A$

$$
A_{k}=k \cdot A=\left\{k \cdot m: m \in A, m=\pi^{-1}(\mathbf{m}) \Rightarrow k \cdot m=\pi^{-1}(k \cdot \mathbf{m})\right\}, k \in R .
$$

Set $A_{k}$ is a dilatation of set $A$ by a non-zero scalar $k \in R$, while it can be geometrically interpreted as image of point set $A$ in the scaling from the centre in the origin $O$ with non-zero coefficient $k$. Minkowski linear combination of two point sets can be then regarded as a certain generalization of Minkowski sum, defined as Minkowski sum of scalar multiples of these two sets.

Definition 4. Minkowski linear sum combination of two point sets $A$ and $B$ in the space $\mathbf{E}^{n}$ is a point set $C$ in the same space $\mathbf{E}^{n}$ determined as

$$
C=k \cdot A \oplus l \cdot B=A_{k} \oplus B_{l}=\{k \cdot a+l \cdot b: a \in A, b \in B\}, k, l \in R .
$$

Visualisation of Minkowski linear sum combinations of two planar curves are presented in Fig. 1, while surface patches in Fig. 2 are linear sum combinations of curves in space.
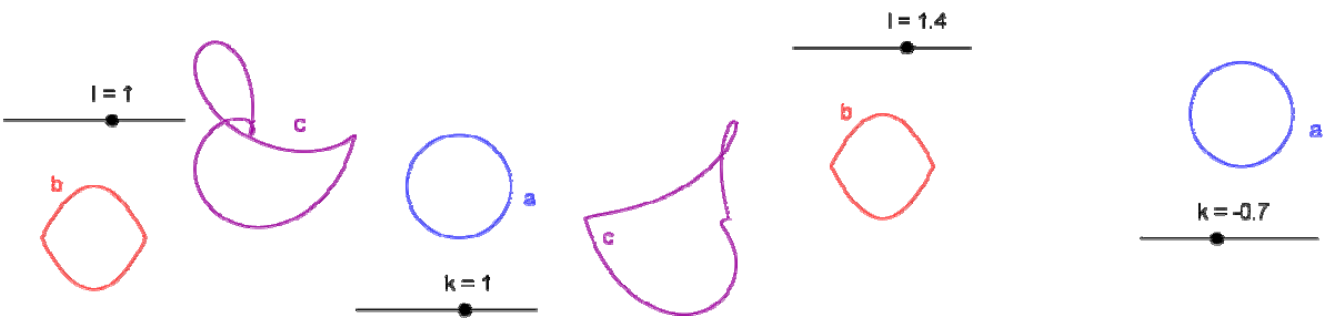

Figure 1: Minkowski linear sum combinations of planar curves.
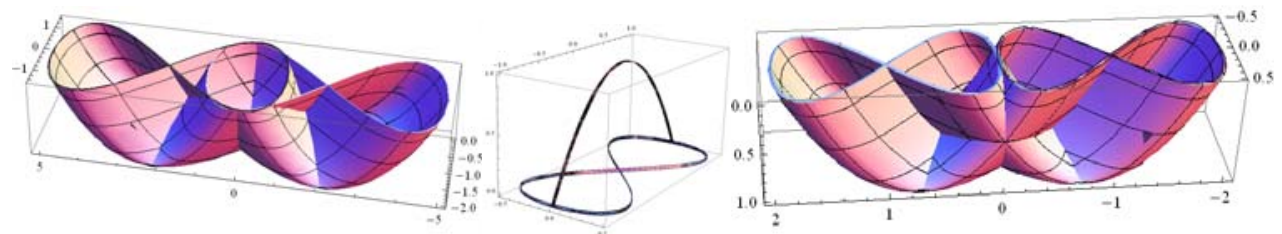

Figure 2: Minkowski linear sum combinations of curves in space. 
Suppose, regular square matrix $\mathbf{M}$ of type $n \times n$ (of rank $n$ ) be given with real entries, representing a linear transformation of the space $\mathbf{E}^{n}$. Matrix multiple of an arbitrary point set $A$ in $\mathbf{E}^{n}$ can be then defined as follows, representing the transformed set $A$ under the transformation represented by matrix $\mathbf{M}$, i.e. image of set $\mathrm{A}$ in the transformation given by matrix $\mathbf{M}$.

Definition 5. Multiple of a point set $A \subset \mathbf{E}^{n}$ by regular square matrix $\mathbf{M}$ of rank $n$ with real entries is a point set $A_{\mathbf{M}} \subset \mathbf{E}^{n}$ with elements in all such points, whose position vectors are images of position vectors of all points from set $A$ under the respective linear transformation determined by matrix $\mathbf{M}$

$$
A_{\mathbf{M}}=A \cdot \mathbf{M}=\left\{m \cdot \mathbf{M}: m \in A, m=\pi^{-1}(\mathbf{m}) \Rightarrow m \cdot \mathbf{M}=\pi^{-1}(\mathbf{m} \cdot \mathbf{M})\right\} .
$$

Set $A_{\mathbf{M}}$ is a transformed set $A$ under the linear transformation determined by regular square matrix $\mathbf{M}$ with real entries, which is the matrix of the system of equations that define the particular linear transformation. Minkowski matrix combination of two point sets $A$ and $B$ can be then regarded as another generalization of Minkowski sum, defined as Minkowski sum of matrix multiples of these two point sets by respective matrices $\mathbf{M}$ and $\mathbf{N}$.

Definition 6. Minkowski matrix sum combination of two point sets $A$ and $B$ in the space $\mathbf{E}^{n}$ is a point set $C$ in the same space $\mathbf{E}^{n}$ determined as

$$
C=A . \mathbf{M} \oplus B . \mathbf{N}=A_{\mathbf{M}} \oplus B_{\mathbf{N}}=\{a \cdot \mathbf{M}+b . \mathbf{N}: a \in A, b \in B\},
$$

where $\mathbf{M}$ and $\mathbf{N}$ are regular square matrices of type $n \times n$ with real entries.

Visualisation of Minkowski matrix sum combinations of the same two planar curves whose Minkowski linear sum combinations are in Fig. 1 are presented in Fig. 3, while surface patches in Fig. 4 are Minkowski matrix sum combinations of curves in space presented in Fig. 2.
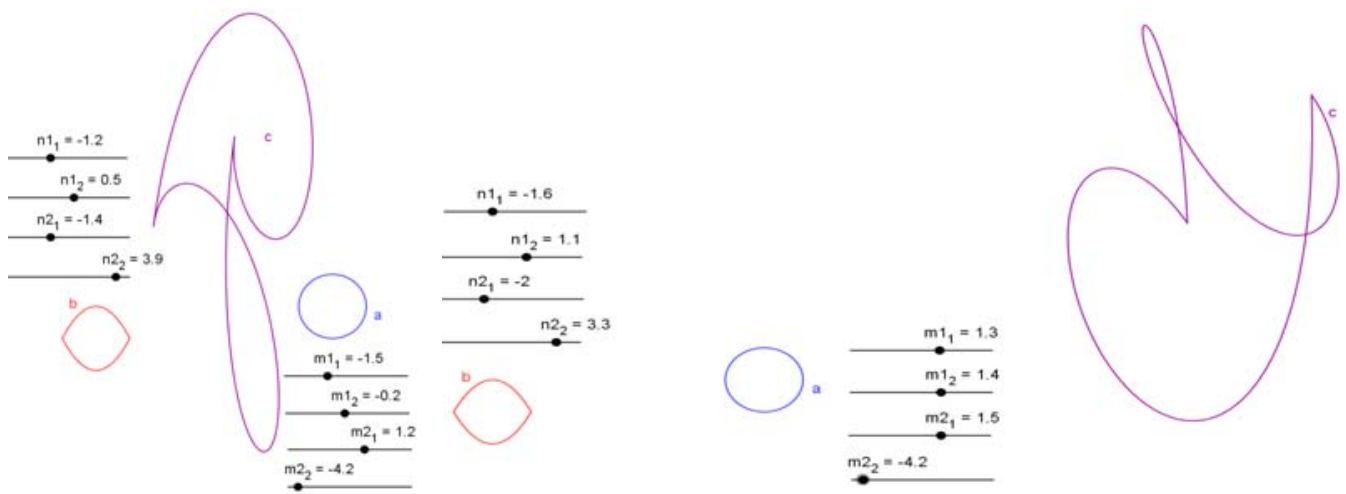

Figure 3: Minkowski matrix sum combinations of planar curves. 

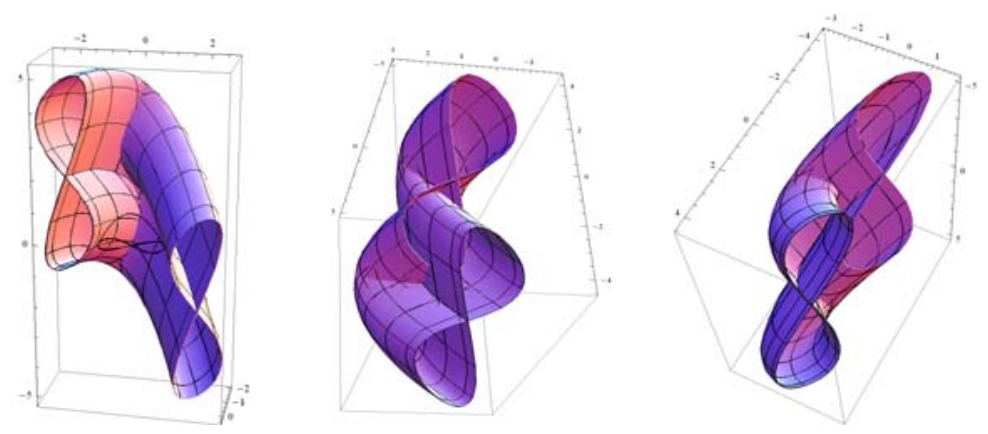

Figure 4: Minkowski matrix sum combinations of space curves.

Definition 7. Product of two points $a, b$ in the space $\mathbf{E}^{n}$ is such point $c \in \mathbf{E}^{p}, p=n(n-1) / 2$, whose position vector from the vector space $W\left(\boldsymbol{E}^{p}\right)$ is the wedge product of position vectors of points $a, b$

$$
a, b \in \mathbf{E}^{n}, \mathbf{a}=\pi(a), \mathbf{b}=\pi(b) \Rightarrow a \wedge b=c \in \mathbf{E}^{p} \Leftrightarrow c=\pi^{-1}(\mathbf{c}), \mathbf{c}=\mathbf{a} \wedge \mathbf{b}
$$

Definition 8. Minkowski product of two different point sets $A$ and $B$ in space $\mathbf{E}^{n}$ is a point set in space $\mathbf{E}^{p}, p=n(n-1) / 2$, whose points are products of all points from $A$ with all points from $B$

$A \otimes B=\{a \wedge b: a \in A, b \in B\}, A \otimes \varnothing=\{0\}$.

Minkowski product combination of two point sets $A$ and $B$ in the space $\mathbf{E}^{n}$ can be defined, for arbitrary real coefficients $k$ and $l$.

Definition 9. Minkowski linear product combination of two different point sets $A$ and $B$ in the space $\mathbf{E}^{n}$ is the point set $C$ in the space $\mathbf{E}^{p}, p=n(n-1) / 2$, defined as Minkowski product of $k$ multiple of set $A$ and $l$-multiple of set $B$

$$
C=k \cdot A \otimes l . B=A_{k} \otimes B_{l}=\{k \cdot a \wedge l \cdot b: a \in A, b \in B\}, k, l \in R .
$$

Minkowski product of two equally parameterized curve segments is a curve segment. Example in Fig. 5 shows Minkowski product of parabolic arc and lemniscate of Bernoulli that are differently parameterized, compared to their Minkowski sum in Fig. 2, on the left. Minkowski product of differently parameterized shamrock curve and versiére that are located in different 3dimensional subspaces of $\mathbf{E}^{4}$ is a spectacular surface patch from the space $\mathbf{E}^{6}$ presented in different 3-D views in Fig. 6, on the left.
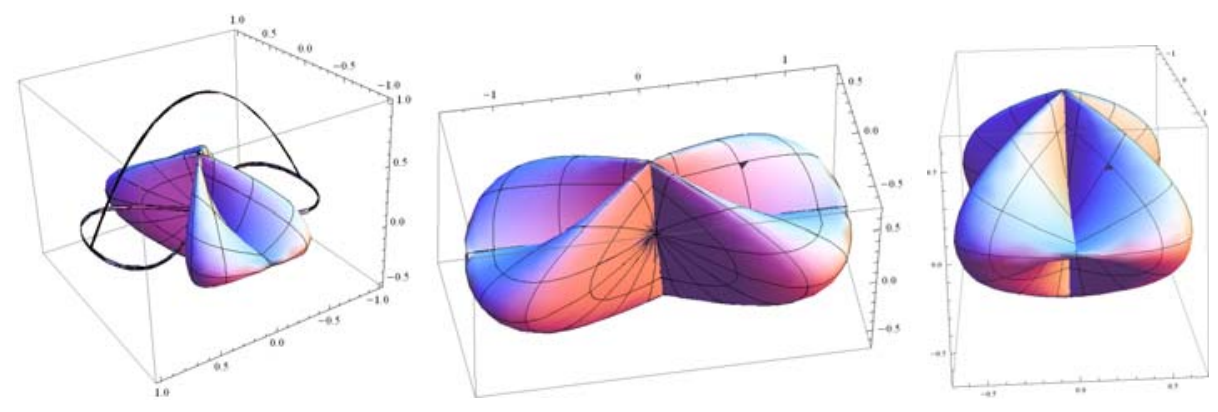
Figure 5: Minkowski product of two curve segments in 3D.
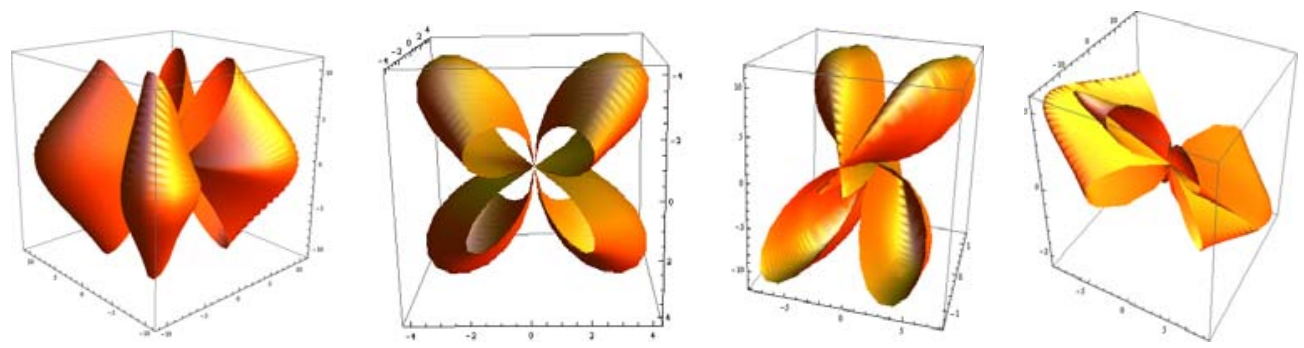

Figure 6: Views of Minkowski matrix product combinations of two curve segments in 4D.

Definition 10. Minkowski matrix product combination of two different point sets $A$ and $B$ in $\mathbf{E}^{n}$ is the point set $C$ in the space $\mathbf{E}^{p}, p=n(n-1) / 2$, defined as Minkowski product of the matrix multiples of sets $A$ and $B$, by square regular matrices $\mathbf{M}$ and $\mathbf{N}$

$$
C=A_{\mathbf{M}} \otimes B_{\mathbf{N}}=A \cdot \mathbf{M} \otimes B \cdot \mathbf{N}=\{a \cdot \mathbf{M} \wedge b \cdot \mathbf{N}: a \in A, b \in B\}
$$

According to the properties of the exterior product the geometric interpretation of Minkowski linear product and Minkowski matrix product combinations can be derived. It can be proved that the resulting set is an image of the Minkowski product of sets $A$ and $B$ respectively modified, i.e. either the original Minkowski product of sets $A$ and $B$ just scaled by the coefficient $k . l$, or image of Minkowski product of sets $A$ and $B$ transformed under the mapping determined by the product of matrices $\mathbf{M}$ and $\mathbf{N}$, therefore set $(A \otimes B)_{\text {M.N. }}$. Illustrations of Minkowski matrix product combinations are in Fig.6, on the right.

\section{CONCLUSIONS}

Theory of Minkowski point set operations can be used for calculation of equidistant to a curve that is trajectory of a rigid body movement in plane or in space. Shape of equidistant curve can be analyzed with respect to possible singular points causing problems to moving body with respect to its dimensions and free space necessary for making turns. This can be a sufficient approach of determining necessary passage left for a robot of certain dimensions that is moving on a given trajectory in a working space with obstacles, as illustrated in Fig. 7.

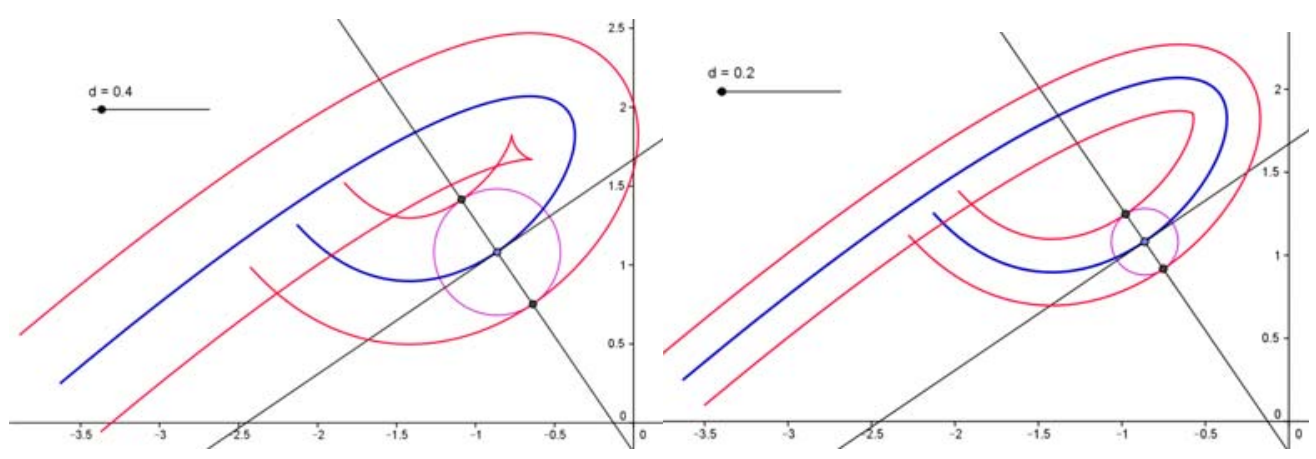

Figure 7: Equidistants to trajectory of a rigid body motion in plane. 


\section{REFERENCES}

[1] Peternel, M., Manhart, F., The Convolution of Paraboloid and a Parametrized Surface. In Journal for Geometry and Graphics 7, 2003, p. 157-171.

[2] Velichová, D., Minkowski Set Operations in Geometric Modelling of Continuous Riemannian Manifolds, In Scientific Proceedings 2009, STU in Bratislava, 2009, SR, ISBN 978-80-227-3326-7, pp. 179-186.

[3] Velichová, D., Multidimensional Riemannian Manifolds as Minkowski Products, In Aplimat - Journal of Applied Mathematics, N²/2012, Volume 4, Slovak University of Technology in Bratislava, 2012, ISSN 1337-6365, pp. 151-158. 